\title{
Temas abordados na consulta de enfermagem: revisão integrativa da literatura
}

\author{
Themes addressed in nursing consultation: integrative literature review \\ Temas tratados en la consulta de enfermería: revisión integradora de la literatura
}

\section{Sherida Karanini Paz de Oliveira', Ana Paula Oliveira Queiroz", Diliane Paiva de Melo Matos", Alline Falconieri de Moura", Francisca Elisângela Teixeira Lima'}

' Universidade Federal do Ceará, Faculdade de Farmácia, Odontologia e Enfermagem, Departamento de Enfermagem, Grupo de Estudos sobre Consulta de Enfermagem. Fortaleza-CE, Brasil.

" Universidade Federal do Ceará, Departamento de Enfermagem. (Graduanda) Fortaleza-CE, Brasil.

Submissão: 19-10-2010 Aprovação: 17-01-2012

\section{RESUMO}

O estudo objetivou analisar os aspectos abordados da consulta de enfermagem (CE) nas publicações científicas. Realizou-se uma revisão integrativa da literatura disponível nos bancos de dados: LILACS, PUBMED, CINAHL e COCHRANE. Foram selecionados 31 artigos que obedeceram aos critérios de inclusão. Os temas mais abordados acerca da CE foram: fatores intervenientes da CE, tempo e custo das consultas, avaliação dos registros de Enfermagem, uso de roteiros de entrevista, comunicação, sistematização da assistência de Enfermagem, significado e importância da CE para promoção da saúde. Conclui-se que diversos aspectos da consulta de Enfermagem estão sendo abordados nos artigos analisados. Contudo, são necessários estudos que comprovem sua eficácia.

Descritores: Enfermagem; Enfermagem baseada em evidências; Cuidados de enfermagem.

\section{ABSTRACT}

The study aimed to analyze the aspects of the nursing consultation (NC) in scientific publications. It was conducted an integrative literature review available in databases: LILACS, PUBMED, CINAHL and COCHRANE. 31 articles were selected that met the inclusion criteria. The themes most addressed on the NC were: factors affecting the NC, time and cost of consultations, assessment of nursing records, use of interview scripts, communication, systematization of nursing care, meaning and importance of the NC to promote health. It was concluded that various aspects of nursing consultation are being addressed in the articles analyzed. However, studies are needed to confirm its efficacy.

Key words: Nursing; Evidence-based nursing; Nursing care.

\section{RESUMEN}

El objetivo fue analizar los aspectos de la consulta de enfermería (CE) en las publicaciones científicas. Se realizó una revisión de la literatura disponible en las bases de datos: LILACS, PUBMED, CINAHL y Cochrane. Fueron seleccionados 31 artículos que cumplían con los criterios de inclusión. Los temas más discutidos acerca de la CE fueron: los factores que afectan a la CE, el tiempo y costo de las consultas, la evaluación de los registros de enfermería, el uso de guiones de entrevista, la comunicación, la sistematización de los cuidados de enfermería y la importancia de la CE para la promoción de la salud. Se concluye que varios aspectos de la consulta de enfermería están siendo tratados en los artículos analizados. Sin embargo, se necesitan estudios para confirmar su eficacia.

Palabras clave: Enfermería; Enfermería basada en la evidencia; Atención de enfermería. 


\section{INTRODUÇÃO}

A Enfermagem preocupa-se constantemente com a meIhoria da assistência, buscando conhecimentos próprios para sistematizar e organizar sua prática e seu processo de cuidar, de modo a favorecer uma assistência baseada não somente na dimensão biológica do ser humano, mas essencialmente na compreensão do homem como sujeito social e o seu processo saúde-doença, seja no âmbito hospitalar ou na saúde coletiva.

Nesse contexto, destaca-se o potencial da consulta de Enfermagem (CE) como estratégia tecnológica de cuidado importante e resolutiva, respaldada por lei, privativa do enfermeiro, e que oferece inúmeras vantagens na assistência prestada, facilitando a promoção da saúde, o diagnóstico e o tratamento precoces, além da prevenção de situações evitáveis.

A entrevista realizada pelo enfermeiro, praticada desde a década de 1920, pode ser considerada uma precursora da consulta de Enfermagem, que só foi instituída em 1968, inicialmente dirigida prioritariamente ao grupo materno-infantil e posteriormente ampliada para todos os grupos. Sua regulamentação ocorreu por meio da lei $n^{\circ} 7498 / 86$ e do decreto $\mathrm{n}^{\circ}$ 94406/87(1).

A consulta de Enfermagem é uma estratégia eficaz para a detecção precoce de desvios de saúde e acompanhamento de medidas instituídas, as quais se dirigem ao bem-estar das pessoas. Viabiliza o trabalho do enfermeiro durante o atendimento ao paciente, facilitando a identificação de problemas e as decisões a serem tomadas. Para tanto, deve ser norteada pela Sistematização da Assistência de Enfermagem (SAE), um método científico com aplicação específica, de modo que o cuidado de Enfermagem seja adequado, individualizado e efetivo ${ }^{(2-3)}$.

Estudos recentes apontam que a consulta de Enfermagem traz benefícios à comunidade e proporciona orientação de medidas favoráveis que visam à abordagem apropriada às necessidades peculiares dos pacientes ${ }^{(4-6)}$.

O número de estudos sobre o tema vem crescendo, visto que os enfermeiros estão buscando consolidar sua profissão como ciência. Nesse esforço, há uma especial atenção à pesquisa, por ser um instrumento que contribui positivamente para o crescimento da Enfermagem e, consequentemente, para a formação profissional dos enfermeiros, no conjunto ou individualmente, ampliando sua visão sobre o processo saúde-doença e possibilitando a melhoria na qualidade da assistência prestada.

O processo de implementação dos resultados de pesquisa passa por algumas etapas, tais como a produção do conhecimento, sua disseminação e utilização, de forma a modificar a prática assistencial de Enfermagem. Desde a década de 70, a literatura internacional de Enfermagem vem mostrando diversos modelos da utilização dos resultados de pesquisa na prática. Nos últimos anos, tanto em eventos científicos, quanto na literatura internacional, educadores, pesquisadores e enfermeiros assistenciais enfatizam a prática baseada em evidências (PBE) que surgiu primeiramente aplicada à Medicina, mas logo se disseminou para todas as áreas da saúde ${ }^{(7)}$.

A PBE encoraja a utilização dos resultados da pesquisa na assistência, fortalecendo ainda mais a pesquisa como forma de aprimoramento das práticas, visto que a grande quantidade de informações no âmbito da saúde, aliada à falta de tempo dos profissionais, determinam a necessidade de elaborar estratégias mais concisas que levem à apropriação dos resultados das pesquisas. Daí a importância da revisão integrativa de literatura ${ }^{(8)}$.

A revisão integrativa pode ser definida como um método em que pesquisas publicadas previamente são sumarizadas e conclusões são estabelecidas considerando o delineamento das investigações examinadas. Isso possibilita a síntese e a análise dos estudos sobre o tema investigado. Permite, também, descrever o conhecimento em seu estado atual; promove o impacto da pesquisa sobre a prática profissional, mantendo os interessados atualizados; e facilita as modificações da prática cotidiana como conseqüência da pesquisa ${ }^{(9)}$.

Acrescenta-se, ainda, que a revisão integrativa tem o potencial para produzir e difundir o conhecimento em Enfermagem, pois resulta em conhecimento fundamentado e uniforme; reduz os obstáculos a sua utilização, tornando a informação mais acessível, uma vez que num único trabalho há a união de várias pesquisas, permitindo assim agilidade da divulgação do conhecimento ${ }^{(8)}$.

Diante da importância da consulta de Enfermagem na assistência ao paciente e por acreditar que o enfermeiro deve se preocupar com a implementação de práticas que ofereçam condições seguras e de qualidade para o desempenho de suas atividades, propusemo-nos a realizar esta investigação, esperando que seus resultados contribuam para divulgação do conhecimento produzido acerca da referida temática.

O estudo foi orientado a responder à seguinte questão: Quais os principais temas e aspectos da consulta de enfermagem estão sendo discutidos nas publicações científicas da área? E seu objetivo foi analisar os temas e aspectos abordados na consulta de enfermagem nas publicações científicas.

\section{MÉTODO}

Trata-se de uma revisão integrativa da literatura referente à produção sobre a consulta de Enfermagem em artigos de periódicos indexados nas seguintes bases de dados: Literatura Latino-Americana e do Caribe de informação em Ciências da Saúde (LILACS), Publicações Médicas (PUBMED), Cummulative Index to Nursing and Allied Health Literature (CINAHL) e COCHRANE.

A busca dos artigos ocorreu no período de dezembro de 2009 a fevereiro de 2010, sendo selecionados 31 artigos que atenderam aos seguintes critérios de inclusão: estar disponível na íntegra nas referidas bases de dados; estar nos idiomas português, inglês ou espanhol; abordar a consulta de Enfermagem e responder a questão norteadora do estudo. Foram utilizados os descritores não-controlados consulta e Enfermagem, e a tradução das palavras associadas em inglês (nursing consultation) e espanhol (consulta de enfermería).

Após a seleção, os artigos foram submetidos a leituras exploratórias e seletivas. A coleta dos dados foi realizada por meio de um formulário com aspectos metodológicos e temáticos dos estudos. A análise dos dados foi fundamentada na literatura pertinente à temática. 
Os aspectos éticos e legais foram respeitados, tendo em vista que foram utilizadas publicações de periódicos nacionais e internacionais, cujos autores foram citados em todos os momentos em que os artigos foram mencionados.

\section{RESULTADOS}

A análise dos estudos selecionados revelou os seguintes temas: fatores intervenientes da consulta de Enfermagem, tempo e custo das consultas, avaliação dos registros de Enfermagem durante as consultas, uso de roteiros de entrevista para norteá-las, comunicação, Sistematização da Assistência de Enfermagem, significado da consulta de Enfermagem para os clientes e importância da consulta para a redução de complicações.

- Fatores intervenientes da consulta de enfermagem à Há diversos fatores que interferem na realização e na qualidade da consulta de Enfermagem, dos quais destacam-se: formação profissional, agendamento de consultas, espaço físico, tempo e alta demanda de clientes.

- Tempo e custo da consulta de Enfermagem à $\mathrm{O}$ cálculo do tempo de duração e o custo das consultas de Enfermagem também foram abordados, com ênfase na importância desses aspectos para estabelecer padrões dos processos de produção, fornecer dados para determinar o custo padrão, estimar o custo de um produto ou serviço e fornecer dados para estudos do balanceamento de estruturas de produção.

- Avaliação dos registros de Enfermagem à Os registros de Enfermagem foram um aspecto organizacional detectado nos artigos analisados, visto que são as fontes primordiais de acesso à informação do cliente.

- Importância do uso de roteiros de entrevistas nas consultas de Enfermagem à Um dos fatores que deve ser considerado para realização da consulta de Enfermagem é o uso de roteiros para norteá-la a fim de impedir mudanças inconsistentes no foco da conversa, devendo o enfermeiro considerar a comunicação verbal e não verbal nas respostas dos pacientes às perguntas.

- Comunicação à Encontrou-se nos estudos que a comunicação é essencial para a realização da consulta de Enfermagem, cabendo ao enfermeiro ter consciência da importância dos comportamentos verbais e não verbais durante a comunicação, reconhecendo emoções, expectativas e estereótipos que possam interferir no atendimento.

- Sistematização da Assistência de Enfermagem (SAE) à Foi referida por diversos estudos como o método norteador para a realização da consulta de Enfermagem.

- Significado da consulta de Enfermagem à Em relação aos significados atribuídos à consulta de Enfermagem pelos pacientes, encontraram-se estudos que identificaram a consulta como a aquisição de informações, satisfação biopsicossocial e espaço de acolhimento.

- Importância da consulta de Enfermagem para a redução de complicações à $\mathrm{A}$ consulta de Enfermagem pode ser realizada para pacientes com os mais diversos tipos de problemas. Contudo, foram identificados estudos que abordaram a importância da consulta de enfermagem com pacientes com problemas gastrintestinais, cardíacos e respiratórios.

\section{DISCUSSÃO}

A consulta de Enfermagem é uma atividade precípua do enfermeiro. Apesar de regulamentada desde a década de 80, ainda é uma tecnologia que não alcançou totalmente a sua vigência nos três níveis de atenção. Entretanto, vem a cada dia sendo empregada tanto pelos profissionais que buscam sua autonomia, quanto a nível acadêmico, para inserir a sua importância desde o início da formação do estudante. No presente estudo, a CE mostrou-se associada a diversos temas, abordados sob diversos aspectos.

\section{Fatores intervenientes da consulta de enfermagem}

A articulação teórico - prática, advinda da formação profissional do enfermeiro, é importante para a aquisição das competências necessárias à realização da consulta de Enfermagem, que possui valor bastante significativo para dar resolubilidade às questões apresentadas pelos pacientes, permitindo atendê-los de maneira holística e integral. Vários autores reiteram a importância da capacitação profissional para um atendimento de qualidade ${ }^{(11-13)}$. Outro fator interveniente é a falta de agendamento das consultas, o que impede a efetivação de um cuidado integral ao paciente. Isso pode ser detectado no estudo de Rios e Vieira ${ }^{(12)}$ que descreveu as condições de trabalho dos enfermeiros na consulta de Enfermagem no pré-natal e constatou que essa não é uma atividade com o agendamento diário sistemático. A via de acesso das gestantes aos enfermeiros aconteceu por dois mecanismos: alívio da demanda, ou seja, drenagem da clientela excedente da consulta médica ou por meio do momento da vacinação, mediante encaminhamento médico ${ }^{(12)}$.

O espaço físico também foi evidenciado como um fator que interfere na qualidade da consulta de Enfermagem. Os ruídos de comunicação, as interrupções, a falta de privacidade comprometem a continuidade da consulta, dificultando a realização de ações educativas ${ }^{(12)}$. Do mesmo modo, o tempo influencia a consulta de enfermagem, uma vez que varia no primeiro atendimento, nas consultas de acompanhamento e no acolhimento à demanda ${ }^{(12)}$. Nas Unidades Básicas de Saúde, a grande demanda de pacientes é considerada uma das maiores dificuldades para a realização da consulta de Enfermagem tal como preconizada pelo Ministério da Saúde, resultando em atendimento mais rápido devido à restrição do tempo ${ }^{(11)}$.

O estudo de Figueiredo e Melo(13) buscou identificar as ações de Enfermagem no acompanhamento do crescimento e desenvolvimento de crianças de Franca-SP e detectou como fatores intervenientes, o espaço físico inadequado (portas abertas, salas com mais de uma atividade e disposição dos móveis), a alta demanda, os diálogos fragmentados, as orientações incompletas e os atendimentos simultâneos. Tais aspectos foram considerados negativos por comprometerem a comunicação, já que em alguns casos não se ouviam as informações, diminuíram as respostas e houve distanciamento entre os sujeitos ${ }^{(13)}$.

\section{Tempo e custo da consulta de enfermagem}

O cálculo do tempo de duração de consultas ou procedimentos nas organizações de saúde é importante para estabelecer padrões dos processos de produção, fornecer dados para 
determinar o custo padrão, estimar o custo de um produto ou serviço novo e fornecer dados para o estudo do balanceamento de estruturas de produção. Assim como a análise dos custos, é importante para avaliar o crescimento quantitativo e financeiro das unidades, conhecer os custos de tratamentos e procedimentos utilizados, negociar preços e remunerações, alocar os recursos de modo eficiente, identificar ineficiências e desperdícios no processo produtivo, dentre outros ${ }^{(14)}$.

A capacidade de produção das consultas pelos profissionais da saúde é preconizada pelo Ministério da Saúde ${ }^{(15)}$, sendo que o enfermeiro deve realizar três consultas por hora e o médico, quatro, não havendo distinções entre a consulta nova e a de seguimento. Contudo, pode haver variações de acordo com convenções das instituições. Portanto, o enfermeiro dispõe em média de 20 minutos para realizar a consulta de Enfermagem.

No entanto, um estudo, desenvolvido no ambulatório do Instituto do Coração do Hospital das Clínicas de São Paulo para estimar o tempo médio da consulta de Enfermagem, encontrou que essa consulta consumiu um tempo médio de aproximadamente 48,91 minutos para as consultas novas, cujo custo do enfermeiro foi de aproximadamente $\mathrm{R} \$ 18,01$. As consultas de seguimento levaram, em média, 22,14 minutos, e o custo do enfermeiro girou em torno de $\mathrm{R} \$ 8,15$. Esses valores são bem superiores à verba repassada pelo Sistema Único de Saúde (SUS) que reembolsa, apenas, R\$2,04 por consulta ${ }^{(14)}$.

Uma vez que o repasse efetuado pelo SUS para os procedimentos não cobre os gastos, não causa surpresa que os gestores dos serviços de saúde estabeleçam intervalos precisos para a realização das consultas, muitas vezes insuficientes para um atendimento de qualidade. Compete aos enfermeiros desenvolver estudos que demonstrem o custo-benefício e a eficácia da consulta de Enfermagem no acompanhamento do paciente, com o intuito de promover e recuperar a sua saúde.

\section{Avaliação dos registros de enfermagem}

O histórico de Enfermagem é realizado na primeira etapa do processo de Enfermagem. Deve ser devidamente registrado no prontuário para fornecer à equipe multiprofissional, dados sobre a evolução do paciente, facilitar a comunicação entre a equipe de saúde, documentar as ações realizadas, testemunhar as ações de enfermagem em processos legais e fornecer subsídios para a conduta diagnóstica e terapêutica. Além disso, possibilita a avaliação dos cuidados de Enfermagem prestados e serve como fonte de consulta para aprendizagem de estudantes e profissionais ${ }^{(3)}$.

Nos registros, o profissional deve identificar o paciente de forma clara e precisa, evitando erros que podem ter consequências irreparáveis, tais como duplicidade ou falta de identificação do paciente, dificultando a assistência individualizada. Em contrapartida, o preenchimento correto dos dados facilita uma ativação no sistema quando uma intervenção imediata é necessária(3).

Registros inadequados podem comprometer a assistên$\mathrm{cia}^{(11)}$. Para facilitar os registros adequados, os coordenadores dos serviços de Enfermagem devem realizar a supervisão e o acompanhamento dos registros realizados pela equipe de
Enfermagem, orientando-a sobre sua importância e necessidade, com ênfase nos seguintes aspectos: registros completos, legíveis, escritos à caneta, sem rasuras ou espaços em branco, assinados e com o número do registro do Conselho Regional de Enfermagem (COREN). Tais condutas podem favorecer a comunicação entre a equipe multidisciplinar de saúde que assiste o paciente.

\section{Importância do uso de roteiros de entrevistas nas consul- tas de enfermagem}

Os roteiros são de grande importância para a organização da consulta de Enfermagem, visto que focam o assunto primordial do problema do paciente, impedindo que faltem informações essenciais para traçar o plano de cuidados ${ }^{(16)}$.

O estudo de Duarte, Ayres e Simonetti(1), realizado em 2008, mostrou que a experiência na utilização dos instrumentos na consulta de Enfermagem é eficiente quanto à forma e conteúdo, uma vez que possibilitam um olhar ampliado do processo saúde-doença, facilitando a atuação do enfermeiro na abordagem integral do paciente.

\section{Comunicação}

A comunicação interpessoal é aquela realizada informalmente na qual há troca de informações verbais e não verbais de forma recíproca. Assim sendo, a comunicação efetiva é um aspecto positivo para o relacionamento entre profissional e paciente, além de proporcionar mais segurança aos pacientes e criar um elo de confiança, o que pode favorecer a adesão ao tratamento. Foi destacado, ainda, que os pacientes percebem a consulta de Enfermagem como diferente da consulta médica, pois favorece o estabelecimento de uma relação mais próxima e individual, marcada pela flexibilidade e informalidade, ressaltando sentimentos como a valorização ${ }^{(17)}$.

A comunicação entre enfermeiro e paciente é denominada comunicação terapêutica porque busca identificar e atender as necessidades de saúde do paciente, criando oportunidades de aprendizagem e permitindo-lhe adquirir conhecimento, segurança e satisfação. Ressaltam, ainda, que a utilização dessa forma de comunicação reflete diretamente na participação do paciente nos esquemas terapêuticos, já que esse necessita de relações de confiança para diminuir o medo, a ansiedade e restabelecer a saúde ${ }^{(11)}$.

Outro aspecto abordado na comunicação é a escuta sensível, a qual possui três tipos de abordagem: científico-clínica, poético-existencial, espiritual-filosófica, utilizadas, respectivamente, para avaliação inicial do paciente, para os fenômenos imprevistos decorrentes do agravamento da doença e para os valores de vida. Para tanto, é necessário que os profissionais saibam ouvir reflexivamente, pois a escuta sensível é imprescindível na avaliação das necessidades de cuidados de Enfermagem $^{(18)}$.

Autores corroboram afirmando que quando o enfermeiro proporciona ao paciente uma participação ativa no seu atendimento, escutando-o com interesse, disposição e favorecendo a sua liberdade de expressão, consegue percebê-lo como um ser único e, dessa forma, planejar a assistência de enfermagem individualizada ${ }^{(12,18-19)}$. 


\section{Sistematização da assistência de Enfermagem}

A sistematização da assistência de enfermagem (SAE) consiste na utilização do processo de Enfermagem como forma de utilizar o método científico nas consultas de Enfermagem. É uma dinâmica de ações inter-relacionadas e sistematizadas que busca uma assistência de qualidade. É composta por cinco fases: histórico de Enfermagem, diagnóstico de Enfermagem, planejamento da assistência, implementação e avaliação de Enfermagem ${ }^{(20)}$. Portanto, muitos estudos fazem referência à SAE como o método norteador da consulta de Enfermagem $^{(1,19,21-23)}$.

Estudo de Cruz e Pimenta ${ }^{(21)}$ propôs um modelo com informações essenciais para uma anamnese de qualidade para pacientes com dor crônica no qual incluem dados de identificação e padrões de respostas humanas, segundo a taxonomia I da North American Nursing Diagnosis Association (NANDA), que já segue os possíveis diagnósticos de Enfermagem. $\mathrm{O}$ instrumento favorece o levantamento de dados de forma completa e o uso dos diagnósticos de Enfermagem como referencial metodológico para o atendimento ambulatorial aos pacientes com dor crônica ${ }^{(21)}$.

O foco central da Enfermagem é o cuidado, com aspectos humanísticos e científicos e é executado por meio do processo de Enfermagem. Autores sugeriram para tal, a criação de um protocolo de levantamento de dados e diagnósticos de Enfermagem, abordando o padrão de respostas humanas preconizadas pela taxonomia I da NANDA, seguido de dados fisiológicos, psicológicos, socioculturais, desenvolvimentais e espirituais ${ }^{(13,15,19)}$.

Um instrumento de consulta de Enfermagem baseado no processo de Enfermagem sugerido por Wanda de Aguiar Horta foi proposto por Duarte, Ayres e Simonetti ${ }^{(1)}$. O referido instrumento foi dividido em quatro etapas: histórico de Enfermagem, levantamento das necessidades humanas de saúde, prescrição e evolução de Enfermagem. Destaca-se que a prescrição de Enfermagem pactuada é uma estratégia para o desenvolvimento da autonomia dos pacientes, podendo favorecer o autocuidado ${ }^{(1)}$.

Felipe, Abreu e Moreira ${ }^{(22)}$ desenvolveram um estudo sobre consulta de Enfermagem a pacientes hipertensos que enfatizou a necessidade do enfermeiro sistematizar suas ações, sendo imperativa a realização do histórico, diagnóstico, planejamento, implementação e evolução, a fim de que seu trabalho e conhecimento conduzam ao repensar contínuo da prática profissional. Para isso, é necessário o desenvolvimento de habilidades específicas em enfermeiros de unidades básicas de saúde para realizarem uma consulta de Enfermagem satisfatória ${ }^{(22)}$

Nesse mesmo estudo(22), foram analisadas as fases desenvolvidas da sistematização da assistência durante a consulta de Enfermagem. Em relação à etapa de levantamento de dados, verificou-se que aspectos essenciais para uma anamnese de qualidade foram abordados de forma parcial e que a ausculta pulmonar ou cardíaca não constava no exame físico. Os diagnósticos, correspondentes à segunda etapa do processo de Enfermagem, tampouco foram estabelecidos. $\mathrm{E}$ com relação aos cuidados implementados, na quarta fase, predominaram a orientação e a educação em saúde. A terceira e quinta etapas não foram mencionadas.

Ao analisar a correlação entre diagnóstico, prescrição e implementação, a pesquisa de Gutiérrez et al. ${ }^{(23)}$ mostrou lacunas na implementação da consulta de Enfermagem, evidenciando a necessidade de melhor preparar os executores dessa função e efetivar ações de cunho interdisciplinar para melhorar os cuidados prestados. Além disso, sugere-se a elaboração de protocolos específicos para cuidados de Enfermagem e o aperfeiçoamento dos seus registros para uma melhor avaliação da qualidade da assistência prestada ${ }^{(23)}$.

\section{Significado da consulta de Enfermagem}

Estudo qualitativo de Araújo e Rosas ${ }^{(24)}$, que buscou compreender o significado da consulta de Enfermagem para o paciente e seu cuidador, constatou que os pacientes procuram a consulta de Enfermagem para orientação da prática do autocuidado. Além disso, diante de uma patologia crônica ou de alta gravidade, os pacientes buscam a consulta de Enfermagem para adquirir informações acerca da doença e do tratamento, visto que uma das ações do enfermeiro é orientar o paciente conforme suas necessidades para promoção e recuperação da saúde ${ }^{(24)}$.

Camargo e Caro $^{(25)}$, em 2009, verificaram que a consulta de Enfermagem baseada no método científico permite a satisfação biopsicossocial dos pacientes, uma vez que enfatiza a atenção a eles dispensada pelo enfermeiro durante a consulta e resulta em sensação de bem-estar após o atendimento.

Já em outro estudo, de Shimizu e Lima ${ }^{(26)}$, desenvolvido com quinze gestantes, para analisar as contribuições e limitações da consulta de Enfermagem no pré-natal, foi constatado que as gestantes reconhecem essa consulta como um espaço de acolhimento, pois possibilita o diálogo, a livre expressão de dúvidas, sentimentos e experiências, estreitando o vínculo entre enfermeiro e paciente.

\section{Importância da consulta de Enfermagem para a redução de complicações}

A importância da consulta de Enfermagem foi destacada para a redução de complicações de pacientes ostomizados, enfatizando o uso do processo de Enfermagem para minimizar os danos do paciente, proporcionando uma maior qualidade de vida(27).

Os cuidados de Enfermagem às pessoas portadoras de ostomia intestinal pretendem estabelecer uma relação efetiva de cuidado, facilitando a reabilitação e estimulando o autocuidado eficiente do paciente. Assim, a assistência de Enfermagem a essa clientela deve englobar as orientações gerais relativas ao tratamento cirúrgico e suas consequências, além de ações específicas de autocuidado, as quais devem ser planejadas e executadas em todas as fases do tratamento ${ }^{(27)}$.

Santos e Oliveira ${ }^{(28)}$ encontraram resultados satisfatórios acerca da consulta de Enfermagem no que concerne à redução de fatores de risco e de diagnósticos de Enfermagem em clientes transplantados cardíacos. No seu papel de educador, o enfermeiro deve considerar a condição sociocultural do paciente para assegurar que os cuidados indicados serão mais 
facilmente aprendidos e realizados, a fim de promover, manter e/ou restaurar a saúde ${ }^{(28)}$.

No Paraná, Bento e Brofman ${ }^{(4)}$ analisaram o impacto da consulta de Enfermagem a pacientes com insuficiência cardíaca e constataram que as consultas, associadas ao tratamento terapêutico adequado, facilitam o cuidado à medida que são realizadas orientações sobre o autocuidado, reduzindo a frequência de internações dos pacientes com insuficiência cardíaca.

No estudo de Bettencourt et al. ${ }^{(29)}$ sobre a consulta de Enfermagem aplicada aos pacientes asmáticos, foram instituídas atividades de educação em saúde tendo como resultados o aumento do conhecimento do paciente sobre sua doença e os cuidados que devem adotar. Isso favoreceu a resolubilidade dos diagnósticos de Enfermagem encontrados nos pacientes em cada consulta subsequente.

Na Saúde Coletiva, o enfermeiro possui um papel importante como mediador entre comunidade e o sistema de saúde. Em diversos estudos foram encontrados resultados positivos para a consulta de Enfermagem ${ }^{(4-6,11-29)}$.

\section{CONSIDERAÇÕES FINAIS}

A consulta de Enfermagem é uma tecnologia leve-dura que funciona melhorando o autocuidado à medida que permite ao paciente desenvolver habilidades próprias para melhorar sua qualidade de vida. É o método no qual o profissional enfermeiro possui completa autonomia para desenvolver estratégias de cuidado abrangentes para a promoção da saúde do paciente, da família ou da comunidade.

Esta revisão integrativa permitiu constatar a diversidade da atuação do enfermeiro nas consultas, sempre corroborando a sua principal função como educador em saúde. Dentre os temas e aspectos abordados nas publicações, verificaram-se facilidades e dificuldades encontradas pelos enfermeiros em realizar uma consulta de Enfermagem adequada, especialmente frente à falta de recursos físicos do ambiente e capacitação profissional referente à aplicação da SAE. Quanto aos aspectos essenciais à consulta, a comunicação destacou-se como ponto de partida para um bom relacionamento entre enfermeiro e paciente.

A redução de complicações também foi mencionada como um ponto positivo da consulta de Enfermagem, haja vista ter sido sempre relatada a diminuição do número de diagnósticos de Enfermagem encontrados no decorrer das consultas, bem como a diminuição da frequência de internações.

Ainda que venha crescendo o número de artigos abordando esse tema, pesquisas adicionais ainda se fazem necessárias para comprovar sua eficácia e produzir evidências de que a consulta de Enfermagem deve ser regulamentada e plenamente estabelecida em todos os níveis de atenção.

\section{REFERÊNCIAS}

1. Duarte MTC, Ayres JA, Simonetti JP. Consulta de enfermagem ao portador de Hanseníase: proposta de um instrumento para aplicação do processo de enfermagem. Rev Bras Enferm 2008;61(n. ${ }^{\circ}$ esp):767-73.

2. Zagonel IPS. Consulta de enfermagem: um modelo de metodologia para o cuidado. In: Carraro TE, Westphalen MEA. Metodologia para assistência de enfermagem: teorização, modelos e subsídios para a prática. Goiânia: $A B$; 2001. p. 41-56.

3. Carvalho ALS, Nobre RNS, Leitão MNA, Vasconcelos CTM, Pinheiro AKB. Avaliação dos registros das consultas de enfermagem em ginecologia. Rev Eletrônica Enferm 2008;10(2):472-83.

4. Bento VFR, Brofman PRS. Impacto da consulta de enfermagem na freqüência de internações em pacientes com insuficiência cardíaca em Curitiba, Paraná. Arq Bras Cardiol 2009;92(6):490-969.

5. Lima YMS, Moura MAV. Consulta de enfermagem pré-natal: a qualidade centrada na satisfação da cliente. R. de Pesq.: cuidado é fundamental 2005; 9( 1/2): 93-9.

6. Cavalcanti ACD, Correia DMS, Queluci GC. A implantação da consulta de enfermagem ao paciente com insuficiência cardíaca. Rev Eletrônica Enferm 2009;11(1):194-9.

7. Caliri MHL, Marziale MHP. A prática de enfermagem baseada em evidências: conceitos e informações disponíveis online. Rev Latinoam Enferm 2000;8(4):103-04.

8. Mendes KDS, Silveira RCCP, Galvão CM. Revisão integrativa: método de pesquisa para incorporação de evidências na saúde e na enfermagem. Texto \& Contexto Enferm 2008;17(4):758-64.

9. Fonseca RMP. Revisão integrativa da pesquisa em enfermagem em centro cirúrgico no brasil: trinta anos após o saep. São Paulo. Dissertação [Mestrado em Enfermagem] Escola de Enfermagem, Universidade de São Paulo; 2008.

10. Santos SMR, Jesus MCP, Amaral AMM, Costa DMN, Arcanjo R. A consulta de enfermagem no contexto da atenção básica de saúde, Juiz de Fora, Minas Gerais. Texto \& Contexto Enferm 2008;17(1): 124-30.

11. Freitas CASL, Silva Neto AV, Ximenes Neto FRV, Albuquerque IMN, Cunha ICK. O. Consulta de enfermagem ao portador de Hanseníase no Território da Estratégia da Saúde da Família: percepções de enfermeiro e pacientes. Rev Bras Enferm 2008; 1(n. ${ }^{\circ}$ esp):757-63.

12. Rios CTF, Vieira NFC. Ações educativas no pré-natal: reflexão sobre a consulta de enfermagem como um espaço para educação em saúde. Ciênc Saúde Coletiva 2007; 12(2):477-86.

13. Figueiredo GLA, Mello DF. A prática de enfermagem na atenção à saúde da criança em unidade básica de saúde. Rev Latino-am. Enfermagem 2003; 11(4): 544-51.

14. Margarido ES, Castilho V. Aferição do tempo e do custo médio do trabalho da enfermeira na consulta de enfermagem. Rev Esc Enferm USP 2006;40(3):427-33.

15. Ministério da Saúde (Brasil). Manual prático para elaboração 
de projetos para unidades de saúde. Espírito Santo.Governo do Estado do Espírito Santo; 2006.

16. Hastings AM, Lennon M, Redsell SA, Cheater FM. Evaluation of a consultation skills workshop using the Consultation Assessment and Improvement Instrument for Nurses. Blackwell Publishing Ltd. Learn Health Soc Care 2003;2(4):202-12.

17. Silva MG, Jesus MCP, Amaral AMM, Costa DMN, Arcanjo RA. A consulta de enfermagem no contexto da comunicação interpessoal: a percepção do cliente. Rev Latinoam Enferma 1998; 6(1): 27-31.

18. Corrêa LA, Santos I, Albuquerque DC. Consulta de enfermagem: Pesquisar/Cuidar através da escuta sensível em uma clínica de insuficiência cardíaca.Online Braz J Nurs (Online) [periódicos na internet];2008;7(1) Disponível em: http://www.objnursing.uff.br//index.php/nursing/ article/view/j.1676-4285.2008.1066/296.

19. (data de acesso não informada pelo autor)

20. Barros SMO, Costa CAR. Consulta de enfermagem a gestantes com anemia ferropriva. Rev Latinoam Enferm 1999;7(4):105-11.

21. Alfaro-Lefreve R. Aplicação do processo de enfermagem: promoção do cuidado colaborativo. $5^{\mathrm{a}}$ ed. Porto Alegre: Artmed; 2005.

22. Cruz DALM, Pimenta CAM. Avaliação do doente com dor crônica em consulta de enfermagem: proposta de instrumento segundo diagnósticos de enfermagem. Rev Latinoam Enferm 1999;7(3):49-62.
23. Felipe GF, Abreu RNDC, Moreira TMM. Aspectos contemplados na consulta de enfermagem ao paciente com hipertensão atendido no Programa Saúde da Família. Rev Esc Enferm USP 2008;42(4): 620-7.

24. Gutiérrez MGR, Adami, N. P.; Castro, R. A. P.; Fonseca, S. M. Natureza e classificação das intervenções de enfermagem em ambulatório de quimioterapia de adultos. Rev Latinoam Enferma 2000; 8(3):33-9.

25. Araújo CRG, Rosas AMMTF. A consulta de enfermagem para clientes e seus cuidadores no setor de radioterapia de hospital universitário. Rev Enferm UERJ 2008;16(3):364-9.

26. Camargo, I. L.; Caro, C. V. C. Comportamientos de cuidado Del anciano e de los profesionales de enfermería: Evidencias para La cualificación profesional. Av Enferm 2009;7(1):48-59.

27. Shimizu HE, Lima MG. As dimensões do cuidado pré-natal na consulta de enfermagem. Rev Bras Enferm 2009;62(3):387-92.

28. Mendonça RS, Valadão $M$, Castro $L$, Camargo TC. A Importância da Consulta de Enfermagem em Pré-operatório de Ostomias Intestinais. Rev Bras Cancerol 2007;53(4):431-5.

29. Santos ZMSA, Oliveira VLM. Consulta de enfermagem ao cliente transplantado cardíaco: impacto das ações educativas em saúde. Rev Bras Enferm 2004;57(6):654-7.

30. Bettencourt ARC, Oliveira MA, Fernandes ALG, Bogossian M. Educação de pacientes com asma: atuação do enfermeiro. J Pneumol 2002;28(4):193-200. 